\title{
Ethylene-increased Accumulation of Fluorescent Lipid-peroxidation Products Detected during Senescence of Parsley by a Newly Developed Method
}

\author{
Shimon Meir, Sonia Philosoph-Hadas, and Nehemia Aharoni \\ Department of Fruit and Vegetable Storage, Agricultural Research Organization, The Volcani \\ Center, Bet Dagan 50250, Israel \\ Additional index words. Petroselinum crispum, malondialdehyde, aldehydes, chlorophyll degradation, leaves
}

\begin{abstract}
A newly developed rapid and convenient method was used for fractionation and analysis of fluorescent compounds (FCs) formed during lipid peroxidation in parsley (Petroselinum crispum Mill.) leaves. These lipofuscinlike FCs [which arise in vivo from reaction of malondialdehyde (MDA) with amino acids] were found to increase during the senescence of detached parsley leaves, following the commencement of chlorophyll degradation and proteolysis. However, accumulation of FCs in response to exogenous ethylene coincided with the onset of chlorophyll loss and proteolysis on day 2 and was accelerated markedly later. Unlike FC accumulation, levels of aldehydes and MDA in control leaves increased more drastically during senescence, but were not affected significantly by exogenous ethylene. The results suggest that the accumulation of FCs in detached parsley leaves exposed to exogenous ethylene is an early senescence-associated process.
\end{abstract}

Lipid peroxidation is a prominent feature of senescence in plant cells and plays a significant role in leaf senescence (Dhindsa et al., 1981; Leshem, 1987; Pauls and Thompson, 1980, 1984; Thomas, 1986). This process yields a mixture of reactive carbonyl compounds via the chain reactions of free radical intermediates (Lillard and Day, 1964). Among these carbonyl products is MDA, measurable by means of a sensitive calorimetric assay (Kosugi and Kikugawa, 1985) that can be used to gauge the relative extent of lipid peroxidation in various systems (Bidlak and Tappel, 1973). Accordingly, MDA has been employed widely and successfully as a positively correlated marker of progressive senescence (Dhindsa, 1982; Dhindsa et al., 1981; Kar, 1986; Paulin and Themistocle, 1988; Pauls and Thompson, 1980; Robert et al., 1980). This highly reactive substance attacks various cellular components by reacting bifunctionally with amino groups of proteins, phospholipids, and nucleic acids to yield conjugated Schiff bases with typical fluorescence properties (Bidlack and Tappel, 1973; Chio and Tappel, 1969; Dillard and Tappel, 1973; Reiss and Tappel, 1973). These chemically heterogeneous FCs are lipofuscin-like pigments characterized by a fluorescence spectrum, whose maxima of excitation and emission fall within rather broad ranges of 350 to $390 \mathrm{~nm}$ and 420 to $470 \mathrm{~nm}$, respectively (Maguire and Haard, 1975). Chloroform-soluble fluorescent products of lipid peroxidation have been discovered in ripening fruits (Maguire and Haard, 1975, 1976) and in senescent leaves (Pauls and Thompson, 1984; Wilhelm and Wilhelmova, 1981). A positive correlation exists between lipid peroxidation and the accumulation of FCs in senescing plant tissues (Pauls and Thompson, 1984; Wilhelm and Wilhelmova, 1981).

This report describes changes in the level of chloroform-soluble FCs during senescence of detached parsley leaves, incu-

Received for publication 10 Sept. 1990. Accepted for publication 30 July 1991 Contribution from the Agricultural Research Organization, The Volcani Center, Bet Dagan, Israel, no. 2973-E, 1990 series. Supported by grant no. US-152588 from BARD (United States-Israel Binational Agricultural Research and Development Fund). The cost of publishing this paper was defrayed in part by the payment of page charges. Under postal regulations, this paper therefore must be hereby marked advertisement solely to indicate this fact. bated with and without ethylene, in comparison with other characteristics of leaf senescence. We also detail a rapid and accurate method for the determination of the level of FCs in senescing detached parsley leaves, using a silica Sep-Pak column. Although silica Sep-Pak columns (Hamilton and Comai, 1984) or bonded phase aminopropyl columns (Kaluzny et al., 1985) were used previously for rapid separation of lipid classes, such columns have not been used for separation of FCs from lipid extracts.

\section{Materials and Methods}

Plant materials and treatments. Experiments were performed with bunches of smooth-leaved parsley that were freshly harvested from the field. Stems (18 cm long) containing only green, fully expanded mature leaves were rinsed with distilled water, distributed at random in groups of 12, and placed in 100-ml flasks containing $50 \mathrm{ml}$ distilled water plus $50 \mu \mathrm{g}$ chloramphenicol/ml. To avoid possible contamination during the incubation period, the stem ends were recut and the solution in the flasks was renewed every second day. Each flask was enclosed in a 2-liter jar that was sealed and connected through fitted inlet and outlet ports to gas cylinders containing either air or air plus ethylene at $10 \mu \mathrm{l} \bullet$ liter $^{-1}$. A flow-through system was used with a flow rate maintained at $50 \mathrm{ml} \bullet \mathrm{min}^{-1}$. Three separate jars were obtained for each sampling period. The parsley bunches in the jars were incubated for 7 days in darkness at $25 \mathrm{C}$. Changes in the levels of their total chlorophyll (Chl), amino acids, and thiobarbituric acid (TBA)-reactants were determined daily with three samples of 10 excised leaves taken from three separate jars. The rest of the leaves in each of the sampled jars were frozen at $-80 \mathrm{C}$, lyophilized, and ground to a fine powder that was stored under nitrogen at $-80 \mathrm{C}$ before extraction and determination of FCs.

Extraction and determination of Chl and amino acids. Chlorophyll and amino acids were extracted from samples of cut leaf

Abbreviations: BHT, butylated hydroxy toluene; FCs, fluorescent compounds; MDA, malondialdehyde; PUFA, polyunsaturated fatty acids; TBA, thiobarbituric acid. 
segments $(0.5 \mathrm{~g})$ by boiling them for $30 \mathrm{~min}$ in test tubes containing $10 \mathrm{ml}$ of $80 \%$ (v/v) ethanol (Gepstein and Thimann, 1981; Philosoph-Hadas et al., 1989). An additional 15-min extraction of the leaf segments in $3 \mathrm{ml}$ of $80 \%$ ethanol was performed, and the ethanolic extracts were combined. Chl was quantitated by transferring 200- $\mu$ l aliquots of the ethanolic extract into $5 \mathrm{ml}$ of $80 \%(\mathrm{v} / \mathrm{v})$ acetone and determining the absorbance at 645 and $663 \mathrm{~nm}$ according to the method of Arnon (1949).

The ethanolic extracts were then evaporated under vacuum at $55 \mathrm{C}$, the residues were dissolved in $2 \mathrm{ml}$ of water, and pigments were removed by addition of $0.5 \mathrm{ml}$ chloroform (Jiao et al., 1986). Amino acid content was. determined in the aqueous solution by the ninhydrin method (Yemm and Cocking, 1954), but adapted to microquantities suitable for enzyme-linked immunosorbent assay (ELISA) plates. The reaction was performed in flat-bottom, 96-well, microtiter plates (Immulon 1; Dynatech, Kloten, Switzerland) in a total volume of $270 \mu \mathrm{l} / \mathrm{well}$, consisting of $10-\mu \mathrm{l}$ sample aliquots plus the original reaction mixture reduced 10 times. A methionine standard curve was included in every plate, and each sample was repeated three or four times. After incubating the plates at $95 \mathrm{C}$ for 20 rein, the absorbance of the colored reaction product was determined at $560 \mathrm{~nm}$ with an automated microplate ELISA Reader (model EL-310; BIO-TEK Instruments, London). This modified procedure enabled a rapid and accurate quantitation of amino acids in many samples.

Extraction and determination of TBA reactants. TBA-reactive substances were extracted from $0.5 \mathrm{~g}$ of tissue by homogenization in $5 \mathrm{ml}$ of solution containing $20 \% \mathrm{TCA}+1.5 \mathrm{~mm}$ EDTA. After a 10-min centrifugation at $10,000 \times \mathrm{g}$, saturated aldehydes in the supernatant were assayed according to the TBA test described by Kosugi and Kikugawa (1985), employing slight modifications. Briefly, $1 \mathrm{ml}$ of $0.67 \%$ TBA was added to $3-\mathrm{ml}$ aliquots of the supernatant and the solution was incubated at 100C for $1 \mathrm{~h}$. The solution was then cooled and centrifuged for $10 \mathrm{~min}$ at $8000 \times \mathrm{g}$. The volume of the resultant supernatant was brought to $10 \mathrm{ml}$ with distilled water and the absorbance of the colored reaction product was determined at $455 \mathrm{~nm}$ for aldehydes (which are products of lipid peroxidation) and at 532 $\mathrm{nm}$ for MDA. For calculations, an extinction coefficient $(\epsilon)$ of $0.457 \times 10^{5} \mathrm{M} \cdot \mathrm{cm}^{-1}$ was used at $455 \mathrm{~nm}$ as an average of the obtained for five aldehydes (1-propanal, 1-butanal, 1-hexanal, 1-heptanal, and 1-propanal-dimethylacetal), and an $\epsilon$ of $1.56 \times 10^{5} \mathrm{M} \cdot \mathrm{cm}^{-1}$ was used for MDA at $532 \mathrm{~nm}$ (Kosugi and Kikugawa, 1985).

Extraction and determination of FCs. Lipofuscin-like fluorescent compounds were extracted from parsley leaves according to the procedure of Fletcher et al. (1973). The dried leaf powder $(0.1 \mathrm{~g})$ was homogenized in $10 \mathrm{ml}$ of 2 chloroform :1 methanol (v/v) solution containing $20 \mathrm{mg}$ butylated hydroxy toluene (BHT)/liter with a Polytron homogenizer (Kinematic, Luzern, Switzerland) for $15 \mathrm{see}$, and warmed for $5 \mathrm{~min}$ at $45 \mathrm{C}$. The homogenate was mixed thoroughly with $10 \mathrm{ml} 0.015 \mathrm{~mm}$ $\mathrm{CaCl}_{2}$ (Folch et al., 1957) and centrifuged (10 min at $10,000 \times$ g) to produce a phase separation. The chloroform phase was processed for removal of interfering pigments as follows: A 400- $\mu$ l aliquot of the chloroform extract (equivalent to $6 \mathrm{mg}$ dry weight) was loaded onto a Sep-Pak silica cartridge (Waters Associates, Milford, Maine) prewashed with $3 \mathrm{ml}$ of analytical chloroform (Frutarom, stabilized with $0.7 \%$ ethanol). Pigments (chlorophylls and carotenoids) were eluted with $6 \mathrm{ml}$ chloroform and discarded. The FCs retained on the column were then eluted with $2.5 \mathrm{ml}$ methanol into a tube containing 20- $\mu$ BHT solution (20 $\mathrm{mg} \cdot \mathrm{ml}^{-1}$ methanol). The level of FCs in the methanol-eluted fractions was determined by recording their fluorescence spectra and intensity (excitation at 300 and $355 \mathrm{~nm}$, emission at 460 $\mathrm{nm}$ ) using an Aminco spectrofluorimeter (model SPF-125; American Instrument Co., Silver Springs, Md.). Standardization was done with a solution of $1 \mu \mathrm{g}$ quinine sulfate/ml (Sigma Israel Chemical Co., Petah-Tikva, Israel) dissolved in $0.1 \mathrm{M}$ $\mathrm{H}_{2} \mathrm{SO}_{4}$. Since all experiments were performed with equal quantities of tissue (6 mg dry weight) and diluted to the same extent, one fluorescence unit was defined as the relative fluorescence measured. Thus, fluorescence intensity was expressed on the basis of units per $6 \mathrm{mg}$ of dry weight (DW). Chloroform extracts stored for 2 weeks at $-20 \mathrm{C}$ gave reproducible results $( \pm 2 \%)$.

All treatments were tested with triplicate bunches, and results are presented as mean \pm SD. Mean separation between control and ethylene-treated leaves for each sampling period is by t test.

\section{Results and Discussion}

Chlorophyll degradation is a visible characteristic metabolic change of leaf senescence. It is usually accompanied or preceded by extensive proteolysis and lipid peroxidation, which in turn are followed by the corresponding accumulation of free amino acids and products of lipid catabolism (Gepstein and Thimann, 1981; Philosoph-Hadas et al., 1989; Thomas, 1986). The first senescence-associated change in any of these components with or without exogenous ethylene was detected after 2 days of darkness, in both control and ethylene-treated bunches (Figs. 1 and 2). A significant promotive effect of ethylene on Chl loss was observed on the 4th day (Fig. 1A), while the ethyleneenhanced accumulations of amino acids (Fig. 1B) and MDA (Fig. 2A) were detected after 3 days of darkness. Significant differences between the aldehyde concentrations of control and ethylene-treated bunches were absent (Fig. 2B), except for MDA

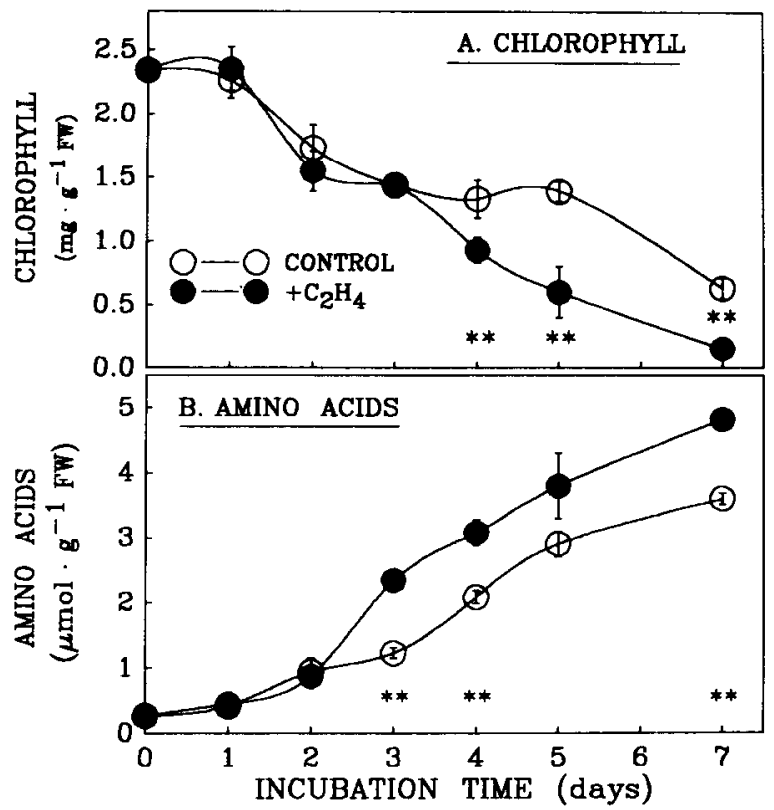

Fig. 1. Effect of exogenous ethylene on chlorophyll (Chl) degradation (A) and amino acid content (B) during dark senescence of parsley leaves. Parsley bunches were kept at $25 \mathrm{C}$ in darkness under air or $10 \mu$ ethylene/liter in a flow-through system. The vertical bars represent SD of three replicates. Mean separation by $t$ test between control and treated leaves for each sampling period are indicated by asterisks, when ${ }^{* *}$ represent significance at $P=0.01$. 

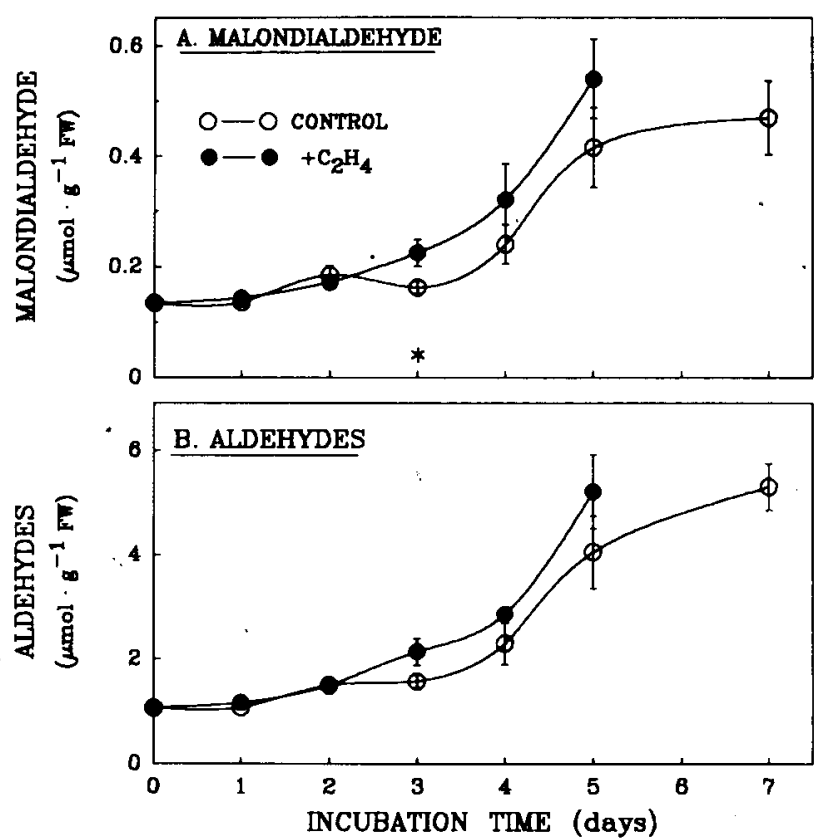

Fig. 2. Effect of exogenous ethylene $\left(10 \mu \mathrm{l} \cdot\right.$ liter $\left.^{-1}\right)$ on changes in levels of MDA (A) and other TBA-reactive aldehydes (B) during dark senecence of parsley leaves. Incubation conditions as detailed in Fig. 1. The vertical bars represent SD of three replicates. Mean separation by $t$ test between control and treated leaves for each sampling period are indicated by asterisks, when $*$ represent significance at $P=0.05$.

levels on day 3 (Fig. 2A). Overall, there was a trend of ethylene enhancement in the level of these lipid oxidation products between days 3 to 5 .

The increase in MDA produced during oxidation of polyunsaturated fatty acids (PUFA) has been widely used as a sign of progressive leaf senescence (Dhindsa et al., 1981; Kar, 1986; Paulin and Themistocle, 1988; Pauls and Thompson, 1980; Robert et al., 1980). However, changes in other aldehydes, which are also TBA-reactive products of PUFA oxidation, have not been used as an indicator of senescence, probably because some of these aldehydes can originate via other pathways. Our results demonstrate that the accumulation patterns of these aldehydes during senescence of control and ethylene-treated parsley bunches (Fig. 2B) are very similar to those of MDA (Fig. 2A). Moreover, after 7 days of senescence, the aldehyde content of control leaves had increased 5.5-fold, while that of MDA increased by only 3.5-fold (Fig. 2). A similar smaller accumulation of MDA relative to other aldehydes was observed during senescence of watercress leaves (Philosoph-Hadas et al., 1989) and other species (unpublished data). This pattern may be attributed to the higher reactivity of MDA, which is bifunctional and can readily react with amino groups to yield other compounds (Bidlack and Tappel, 1973; Chio and Tappel, 1969; Dillard and Tappel, 1973). Our results suggest that although TBA-reactive aldehydes are not exclusively products of lipid oxidation, their accumulation may provide,' in many cases, a more sensitive measure of leaf senescence than MDA increase.

Because MDA is quite reactive, it was of interest to examine the formation of MDA-products as an indicator of leaf senescence. These MDA products are the FCS, which have been found to accumulate in various senescing plant tissues (Maguire and Haard, 1975, 1976; Pauls and Thompson, 1984; Wilhelm and Wilhelmova, 1981) and are extractable with lipid solvents
(Fletcher et al., 1973). However, Duggelin et al. (1988a, 1988b) have recently reported water-soluble FCs present in extracts of senescing monocot leaves [meadow fescue (Festuca pratensis Huds.) and barley (Her-dewn vulgare L.)]. These water-soluble FCs, which showed spectral properties similar to those of the chloroform-soluble FCs, were hypothesized to originate from chlorophyll catabolism rather than from peroxidation of PUFA. Accumulation of FCS in senescing parsley leaves was assayed using the chloroform phase. The fluorescence spectra of FCs extracted from parsley leaves senescing in the absence (Fig. 3A) or presence (Fig. 3B) of exogenous ethylene differ in their magnitude, showing a higher level in response to ethylene. Nevertheless, the excitation spectra of FCs from both treated or untreated parsley leaves show a peak with a maximum at $300 \mathrm{~nm}$ (Fig. 3 ), in addition to the excitation peak ranging from 350 to 380 $\mathrm{nm}$ observed previously in lipid extracts of ripening fruits (Maguire and Haard, 1975, 1976) and senescing cotyledons (Pauls and Thompson, 1984). Wilhelm and Wilhelmova (1981) have also reported two or three excitation maxima at the 300- to 380$\mathrm{nm}$ region present in lipid extracts of chloroplasts isolated from 8- and 36-day-old leaves of Phaseol us vulgaris L. Recently, Wang et al. (1988) obtained a fluorescence spectrum of FCs extracted from rice (Oryza sativa L.) leaves almost identical to that shown here for parsley leaves (Fig. 3), with excitation maxima at 310 and $360 \mathrm{~nm}$. The emission spectra of FCs from parsley leaves, with a broad peak between 455 and $490 \mathrm{~nm}$ (Fig. 3), resemble those of FCs obtained from other species (Maguire and Haard, 1975, 1976; Pauls and Thompson, 1984; Wang et al., 1988; Wilhelm and Wilhelmova, 1981).

The intensity of fluorescence in the parsley lipid extracts increased with the progress of senescence (Figs. 3 and 4). A 2fold increase in intensity was detected during senescence of control leaves (Fig. 4), suggesting a relatively moderate rate of change in this process, as compared with the more pronounced

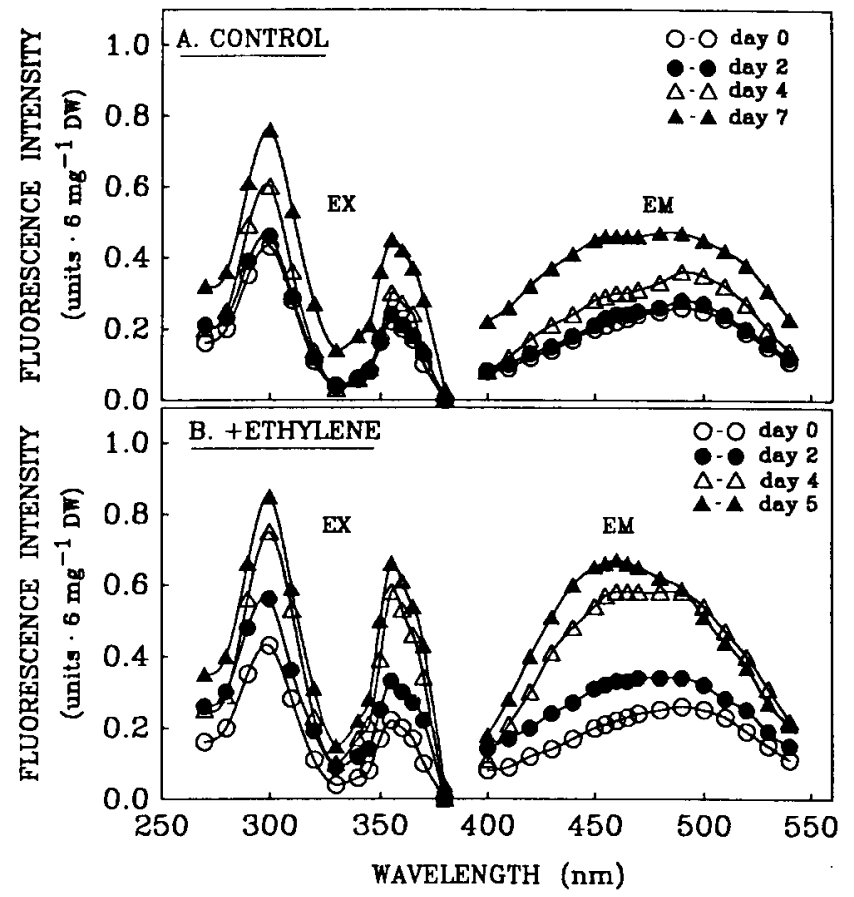

Fig. 3. Representative excitation (EX) and emission (EM) spectra for lipid extracts isolated from control (A) and ethylene-treated (B) parsley leaves during dark-induced senescence. Incubation conditions as detailed in Fig. 1. 


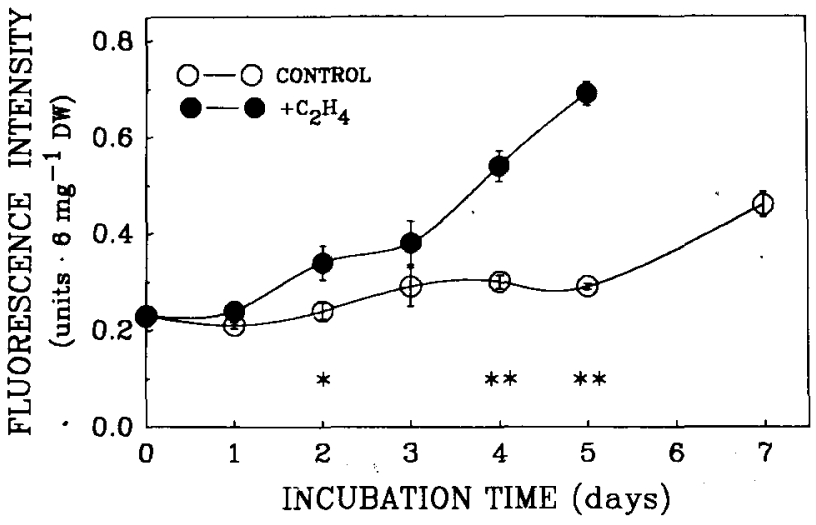

Fig. 4. Effect of exogenous ethylene on the fluorescence intensity (excitation, $355 \mathrm{~nm}$; emission, $460 \mathrm{~nm}$ ) of lipid extracts isolated from senescing parsley leaves. Experimental conditions were detailed in Figs. 1 and 3. The vertical bars represent SD of three replicates. Mean separation by $t$ test between control and treated leaves for each sampling period are indicated by asterisks, when * and ** represent significance at $P=0.05$ and 0.01 , respectively.

Table 1. Effect of ethylene on the daily increase in the relative fluorescence intensity of lipid extracts isolated from parsley leaves.

\begin{tabular}{|c|c|c|c|c|}
\hline \multirow{3}{*}{$\begin{array}{c}\text { Incubation } \\
\text { time } \\
\text { (days) }\end{array}$} & \multicolumn{4}{|c|}{ Ratio of fluorescence intensity ${ }^{2}$} \\
\hline & \multicolumn{2}{|c|}{ Excitation at $300 \mathrm{~nm}$} & \multicolumn{2}{|c|}{ Excitation at $355 \mathrm{~nm}$} \\
\hline & Control & Ethylene & Control & Ethylene \\
\hline$o$ & 1.00 & 1.00 & 1.00 & 1.00 \\
\hline 1 & 1.04 & 1.07 & 0.91 & 1.04 \\
\hline 2 & 1.03 & 1.50 & 1.04 & 1.48 \\
\hline 3 & 1.25 & 1.45 & 1.26 & 1.65 \\
\hline 4 & 1.25 & 1.61 & 1.30 & 2.35 \\
\hline 5 & 1.25 & 2.00 & 1.26 & 3.00 \\
\hline 7 & 1.82 & n.d. & 2.00 & n.d. \\
\hline
\end{tabular}

'Incubation conditions were as described in Fig. 1. Values represent ratio of the relative fluorescence intensity (excitation $=\mathrm{Ex}, 300$ and $355 \mathrm{~nm}$; emission $=\mathrm{Em}, 460 \mathrm{~nm}$ ) of lipids extracted after 1 to 7 days in darkness to the relative fluorescence intensity at zero-time. Data for $E x=355 \mathrm{~nm}$ were taken from Fig. 4. Not detectable $=$ n.d.

changes observed in the other senescence-associated characteristics (Figs. 1 and 2). The senescence-associated changes in Chl degradation (Fig. 1A) and proteolysis (Fig. 1B) could already be detected in control leaves on the 2nd day of incubation and thus preceded by 1 day the commencement of FCs accumulation (Fig. 4). FC accumulation preceded by 1 day the increase of TBA-reactive compounds in control leaves (Fig. 2), an increase that became significant only on the 4th day of incubation. As shown in Fig. 3B, FCs accumulated more rapidly in response to exogenous ethylene, and significant differences in fluorescence intensity between control and ethylene-treated leaves were already found on the 2nd day of incubation (Fig. 4). These ethylene-induced differences in fluorescence intensity increased further with the progress of senescence (Fig. 4), unlike the nonsignificant ethylene-induced differences in TBA-reactive compounds (Fig. 2).

The ethylene-induced fluorescence intensity (emission at 460 $\mathrm{nm}$ ) on day 5 had increased by 3 -fold with excitation at 355 $\mathrm{nm}$, but only 2 -fold with excitation at $300 \mathrm{~nm}$ (Table 1). Thus, a $50 \%$ difference in fluorescence intensity was detected between excitation at 355 and $300 \mathrm{~nm}$, while in control leaves the changes in fluorescent intensity at both excitation wavelengths were similar throughout incubation $(<10 \%)$. These differences in exci- tation spectra may indicate that, in ethylene-treated leaves, reactions of MDA yield a different composition of FCs. Further support for such occurrence of chemically distinct FCs is derived from the fact that the emission spectra with excitation at $300 \mathrm{~nm}$ showed a peak at $355 \mathrm{~nm}$ (data not shown), unlike the emission spectra with excitation at $355 \mathrm{~nm}$, which peaked at $460 \mathrm{~nm}$. Since the 355-rim emission peak is also an excitation one, only the data referring to emission at $460 \mathrm{~nm}$ were presented in Table 1.

The progression of lipid peroxidation is measured in the present study by two sequential reactions: formation of TBA-reactive aldehydes resulting from oxidation of PUFA (Fig. 2) and reaction of one of these aldehydes (MDA) with amino groups to form FCS (Figs. 3 and 4). Dillard and Tappel (1973) found that fluorescence in the lipid fraction was 10 to 100 times more sensitive as a measure of lipid peroxidative damage than the calorimetric MDA assay. In our study, the accumulation of products from both reactions was found to be positively correlated with the progress of senescence, but differed with response to ethylene. Two possible explanations for these differences are suggested: 1) MDA formation (Fig. 2A) seemed to be responsive to ethylene, as was formation of FCs (Fig. 4), but its accumulation was not pronounced on the 2nd day of incubation, presumably because the ethylene-induced increment in MDA level may have been eliminated by its subsequent conversion to FCs; and 2) the process of FCs formation from MDA maybe more affected by exogenous ethylene than MDA formation.

The increased fluorescence of lipid extracts from senescing . tissues can be interpreted as reflecting peroxidative damage (Pauls and Thompson, 1980, 1984). In this respect, reactivity of MDA may be a factor that enhances senescence due to its bifunctional binding to 1 -amino groups of proteins (Crawford et al., 1967), which results in inhibition of various enzymes (Chio and Tappel, 1969). Moreover, MDA can also react with DNA by crosslinking its nucleotides and consequently forming fluorescent products (Reiss and Tappel, 1973). Hence, in addition to being positively correlated with progressive senescence, MDA may serve as a subsequent stimulant of the process due to its effect on senescence-associated enzymes and proteins (Chio and Tappel, 1969; Crawford et al., 1967; Dhindsa, 1982; Reiss and Tappel, 1973).

The importance of this study lies in the development of a rapid and convenient method for determination of FCs in leaves, which, we believe, can be applied successfully to other green tissues. With this method we demonstrated that accumulation of fluorescent lipid-peroxidation products can be regarded as one of the earliest detectable processes that responds to exogenous ethylene during the senescence of detached parsley leaves.

\section{Literature Cited}

Arnon, D.I. 1949. Copper enzymes in isolated chloroplasts. Polyphenol-oxidase in Beta vulgaris. Plant Physiol. 24:1-15.

Bidlack, W.R. and A.L. Tappel. 1973. Fluorescent products of phospholipids during lipid peroxidation. Lipids 8:203-206.

Chio, K.S. and A.L. Tappel. 1969. Inactivation of ribonuclease and other enzymes by peroxidizing lipids and by malonaldehyde. Biochemistry 8:2827-2832.

Crawford, D. L., T.C. Yu, and R.O. Sinnhuber. 1967. Reaction of malonaldehyde with protein. J. Food Sci. 32:332-335.

Dhindsa, R.S. 1982. Inhibition of protein synthesis by products of lipid peroxidation. Photochemistry 21:309-313.

Dhindsa, R. S., P. Plumb-Dhindsa, and T.A. Thorpe. 1981. Leaf senescence: Correlated with increased levels of membrane permeabil- 
ity and lipid peroxidation and decreased levels of superoxide dismutase and catalase. J. Expt. Bet. 32:93-101.

Dillard, C.J. and A.L. Tappel. 1973. Fluorescent products from reaction of peroxidizing polyunsaturated fatty acids with phosphatidyl ethanol-amine and phenylalanine. Lipids 8:183-189.

Duggelin, T., K. Bortlik, H. Gut, P. Matile, and H. Thomas. 1988a. Leaf senescence in Festuca pratensis: Accumulation of lipofuscinlike compounds. Physiol. Plant. 74:131-136.

Duggelin, T., M. Schellenberg, K. Bortlik, and P. Matile. 1988b. Vacuolar location of lipofuscin- and proline-like compounds in senescent barley leaves. J. Plant Physiol. 133:492-497.

Fletcher, B. L., C.J. Dillard, and A.L. Tappel. 1973. Measurement of fluorescent lipid peroxidation products in biological systems and tissues. Anal. Biochem. 52:1-9.

Folch, J., M. Lees, and G.H. Sloane-Stanley. 1957. A simple method for isolation and purification of total lipids from animal tissues. J. Biol. Chem. 226:497-509.

Gepstein, S. and K.V. Thimann. 1981. The role of ethylene in the senescence of oat leaves. Plant Physiol. 68:349-354.

Hamilton, J. G. and K. Comai. 1984. Separation of neutral lipids and fatty acids by high-performance liquid chromatography using low wavelength ultraviolet detection. J. Lipid Res. 25:1142-1148.

Jiao, X. Z., S. Philosoph-Hadas, L. Y. Su, and S. F. Yang. 1986. The conversion of 1-(malonylamino) cyclopropane-l-carboxylic acid to 1amino cyclopropane-1-carboxylic acid in plant tissues. Plant Physiol. 81:637-641.

Kaluzny, M.A., L.A. Duncan, M.V. Merritt, and D. E. Epps. 1985. Rapid separation of lipid classes in high yield and purity using bonded phase columns. J. Lipid Res. 26:135-140.

Kar, M. 1986. The effect of photoperiod on chlorophyll loss and lipid peroxidation in excised senescing rice leaves. J. Plant Physiol. 123:389-393.

Kosugi, H. and K. Kikugawa. 1985. Thiobarbituric acid reaction of aldehydes andoxidized lipids in glacial acetic acid. Lipids20:915920.

Leshem, Y.Y. 1987. Membrane phospholipid catabolism and $\mathrm{Ca}^{2+}$ activity in control of senescence. Physiol Plant. 69:551-559.
Lillard, D.A. and E.A. Day. 1964. Degradation of monocarbonyls from autoxidizing lipids. J. Amer. Oil Chem. Soc. 41:549-554.

Maguire, Y.P. and N.F. Haard. 1975. Fluorescent product accumulation in ripening fruit. Nature 258:599-600.

Maguire, Y. P. and N. F. Haard. 1976. Isolation of lipofuscin-like fluorescent products from ripening banana fruit. J. Food Sci. 41:551554.

Paulin, A. and C. Themistocle. 1988. Influence des déficits hydriques sur la degradation et la biosynthèse des lipides membranaires au tours de la senescence de fleurs d'oeillet (Dianthus caryophyllus L. cv. Ember) cueillies normalement ou en juene bouton. Compt. Rend Acad. Sci. (Paris) serie 111.306:307-312.

Pauls, K.P. and J.E. Thompson. 1980. In vitro simulation of senescence-related membrane damage by ozone-induced peroxidation. Nature 283:504-506.

Pauls, K. P. and J. E. Thompson. 1984. Evidence for the accumulation of peroxidized lipids in membranes of senescing cotyledons. Plant Physiol. 75:1152-1157.

Philosoph-Hadas, S., E. Pesis, S. Meir, A. Reuveni, and N. Aharoni. 1989. Ethylene-enhanced senescence of leafy vegetables and fresh herbs. Acts Hort. 258:37-45.

Reiss, U. and A. L. Tappel. 1973. Fluorescent product formation and changes in structure of DNA reacted with peroxidizing arachidonic acid. Lipids 8:199-202.

Robert, R., C. Stewart, and J.D. Bewley. 1980. Lipid peroxidation associated with accelerated aging of soybean axes. Plant Physiol. 65:245-248

Thomas, H. 1986. The role of polyunsaturated fatty acids in senescence. J. Plant Physiol. 123:97-105.

Wang, W., G.C. Sun, and Z.F. Lin. 1988. Lipofuscin-like pigments inchloroplasts and effects of malondialdehyde and $\alpha$-tocopherolon its accumulation. Plant Physiol. Commun. 3:31-34.

Wilhelm, J. and N. Wilhelmova. 1981. Accumulation of lipofuscinlike pigments in chloroplasts from senescent leaves of Phaseolus vulgaris. Photosynthetic 15:55-60.

Yemm, E.W. and E.C. Cocking. 1954. The determination of amino acids with ninhydrin. Analyst 80:209-213. 